\title{
Spontaneous parity breaking in three-dimensional non-Abelian gauge theory
}

\author{
Hsien-Chung Kao \\ Institute of Physics, Academia Sinica, Taipei, Taiwan \\ Yeong-Chuan Kao \\ Department of Physics, National Taiwan University Taipei, Taiwan \\ Yu-Li Lee \\ Department of Physics, National Taiwan University Taipei, Taiwan
}

(Received 15 September 1995)

\begin{abstract}
We study the parity-odd part of the gauge field two-point function in the effective action in threedimensional non-Abelian gauge theory with both Higgs fields and the Chern-Simons term. It is shown that, contrary to a previous proposal, there is no hint of spontaneous parity breakdown up to one-loop level, if care is taken to turn off the Chern-Simons coefficient before expanding the effective action into local terms. [S0556-2821(96)04014-3]
\end{abstract}

PACS number(s): 11.30.Er, 11.10.Kk, 11.30.Qc, 12.38.Bx

A most interesting feature of three-dimensional (Abelian and non-Abelian) gauge theories is that the Lagrangians admit a term, the parity-odd Chern-Simons term [1], which renders the gauge bosons massive without damaging the invariance under the usual topologically trivial gauge transformations. Moreover, in the non-Abelian case, because of the existence of the topologically nontrivial gauge transformations, the coefficient of the Chern-Simons term must be quantized if the theory is not to suffer from inconsistencies [2]. The reason for the quantization condition here is exactly the same as that for Dirac's quantization condition on the magnetic monopole charge. There have been extensive studies on the quantum corrections to the coefficient of the Chern-Simons term in the Yang-Mills-Chern-Simons theory $[3,4]$. It was found that the renormalized coefficient received a one-loop contribution that is, up to a numerical constant, equal to $N$ [for $\mathrm{SU}(N)$ gauge group] times the signature of the bare coefficient. It seems that the one-loop contribution, hence the renormalized coefficient, does not vanish even if the bare coefficient is set to zero. This observation inspired Khlebnikov and Shaposhnikov [5] to propose that parity may be broken spontaneously in three-dimensional non-Abelian gauge theories. (The situation is completely analogous to the study of spontaneous magnetization in magnetic systems to which one first adds an infinitesimal external magnetic field and observes whether an induced magnetization occurs after the external magnetic field is switched off.)

In extracting the one-loop contribution to the local ChernSimons term, we have to do (external) momentum expansion on the parity-odd part of the gauge field two-point function in the effective action that is a nonlocal functional in position space. A subtle point unnoticed in previous studies on spontaneous parity breakdown (SPB) is that the two procedures (doing momentum expansion and setting the bare ChernSimons coefficient to zero) do not commute. If we turn off the bare Chern-Simons term first, which is the correct choice in the study of SPB, we would find that the one-loop contribution to the parity-odd part of the gauge field two-point function vanishes, as will be shown below. On the other hand, as explained above, setting the external momentum to zero first would leave behind an induced Chern-Simons term even if the bare Chern-Simons coefficient is then put to zero. Therefore, barring nonperturbative effects, the claim that SPB occurs in three-dimensional non-Abelian gauge theories is unwarranted.

To demonstrate our point, we shall present the one-loop correction to the parity-odd part of the gauge field two-point function in the $\mathrm{SU}(N)$ Yang-Mills-Chern-Simons theory with Higgs fields in the fundamental representation. We study both the case where the gauge symmetry is unbroken and the case where $\operatorname{SU}(N)$ is broken down spontaneously to $\mathrm{SU}(N-1)$. The theory is chosen because it is a generalization of the model studied by Khlebnikov and Shaposhnikov [5] in which they contrast SPB with spontaneous gauge symmetry breakdown. Other groups [6,7] have also studied the theory for a variety of motivations, including trying to understand whether the quantization condition on the ChernSimons coefficient is respected by the quantum fluctuations or not. To obtain the gauge field two-point function, it is most appropriate to employ the background field Landau gauge whose conceptual and computational advantages are amply explained in Refs. [8] and [9].

The theory we study is defined by the following Lagrangian in the three-dimensional Euclidean spacetime:

$$
\mathcal{L}=\mathcal{L}_{v}+\mathcal{L}_{m}+\mathcal{L}_{\mathrm{GF}}+\mathcal{L}_{\mathrm{gh}}
$$

where $\mathcal{L}_{v}$ is the gauge field Lagrangian

$$
\begin{aligned}
& \mathcal{L}_{v}= \frac{-1}{2 g^{2}} \operatorname{Tr}\left(G_{\mu \nu} G_{\mu \nu}\right) \\
&-\frac{i \kappa}{4 \pi} \varepsilon_{\mu \nu \lambda} \operatorname{Tr}\left(G_{\mu} \partial_{\nu} G_{\lambda}-\frac{2 i}{3} G_{\mu} G_{\nu} G_{\lambda}\right), \\
& G_{\mu \nu}=\partial_{\mu} G_{\nu}-\partial_{\nu} G_{\mu}-i\left[G_{\mu}, G_{\nu}\right] .
\end{aligned}
$$

In the covariant background field gauge we are using, $G_{\mu}$ is written as $A_{\mu}+Q_{\mu}$ where $A_{\mu}$ represent the background and 
$Q_{\mu}$ are the quantum fluctuations to be integrated over. The $\kappa$ in Eq. (2), usually denoted as $4 \pi \mathrm{m} / \mathrm{g}^{2}$ where $|\mathrm{m}|$ is the gauge-invariant mass for gauge bosons [3], should take integral value for the consistence of the theory. The Higgs field Lagrangian $\mathcal{L}_{m}$ is

$$
\mathcal{L}_{m}=\left(\partial_{\mu} \Phi-i G_{\mu} \Phi\right)^{\dagger}\left(\partial_{\mu} \Phi-i G_{\mu} \Phi\right)+\lambda\left(\Phi^{\dagger} \Phi-\frac{v^{2}}{2}\right)^{2},
$$

with

$$
\Phi=V+\widetilde{\phi}, \quad V=1 / \sqrt{2}\left(\begin{array}{c}
0 \\
0 \\
\vdots \\
v
\end{array}\right) .
$$

It is straightforward to write down the gauge-fixing term $\mathcal{L}_{\mathrm{GF}}$ in the background field $R_{\xi}$ gauge:

$$
\begin{gathered}
\mathcal{L}_{\mathrm{GF}}=\frac{\left(f^{a} f^{a}\right)}{2 \xi}, \\
f^{a}=\partial_{\mu} Q_{\mu}^{a}+\left(A_{\mu} \times Q_{\mu}\right)^{a}-i \xi\left(\widetilde{\phi}^{\dagger} T^{a} V-V^{\dagger} T^{a} \widetilde{\phi}\right),
\end{gathered}
$$

where $(E \times F)_{a} \equiv f_{a b c} E_{b} F_{c} . \quad \mathcal{L}_{\mathrm{GH}}$ is the corresponding Faddeev-Popov ghost term:

$$
\begin{aligned}
\mathcal{L}_{\mathrm{GH}}= & \partial_{\mu} \bar{\eta}_{a} \partial_{\mu} \eta_{a}-\partial_{\mu} \bar{\eta} \cdot\left[\eta \times\left(A_{\mu}+Q_{\mu}\right)\right]+\bar{\eta} \cdot\left(\partial_{\mu} \eta \times A_{\mu}\right) \\
& +\left(\bar{\eta} \times A_{\mu}\right) \cdot\left[\eta \times\left(A_{\mu}+Q_{\mu}\right)\right]+\xi \bar{\eta}_{a} \eta_{b} V^{\dagger}\left\{T^{a}, T^{b}\right\} V \\
& +\xi \bar{\eta}_{a} \eta_{b}\left(\widetilde{\phi}^{\dagger} T^{a} T^{b} V+V^{\dagger} T^{a} T^{b} \widetilde{\phi}\right) .
\end{aligned}
$$

Here, $T^{a}$ are the $\mathrm{SU}(N)$ generators in the fundamental representation satisfying $\left[T^{a}, T^{b}\right]=i f^{a b c} T^{c}$, with $a=1, \ldots, N^{2}-1$.

To diagonalize the quadratic part of the gauge fields, we introduce the following basis, which can be grouped into three categories [10]: (1) generators for the unbroken SU $(N-1), S^{m}$, whose elements in the $N$ th column and row are zero; (2) broken off diagonal generators $R_{ \pm}^{p}$, with $p=1, \ldots, N-1 . \quad R_{-}^{p}=\left(R_{+}^{p}\right)^{\dagger}, \quad$ and $\left(R_{+}^{p}\right)_{\alpha \beta}$ $=\delta_{p \alpha} \delta_{N \beta}$; (3) a broken diagonal generators $T^{D=}$ $\operatorname{diag}(1, \ldots, 1,-\sqrt{(N-1) / 2 N})$. They satisfy the commutation relation

$$
\begin{gathered}
{\left[S^{l}, S^{m}\right]=i f^{l m n} S^{n},} \\
{\left[S^{m}, R_{ \pm}^{p}\right]= \pm\left(S^{m}\right)_{q p} R_{ \pm}^{q},} \\
{\left[S^{m}, T^{D}\right]=0,}
\end{gathered}
$$

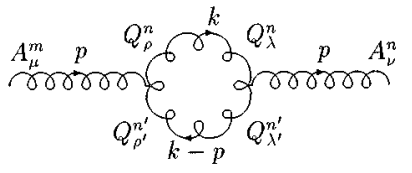

(a)

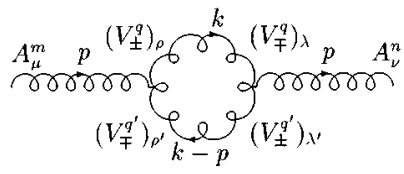

(b)
FIG. 1. The one-loop diagrams that contribute to the parity-odd part of the vacuum polarization in the unbroken sector. (a) involves internal unbroken gluon propagator $\Delta_{\mu \nu}(k)$, while (b) involves internal broken gluon propagator $\Delta_{\mu \nu}^{W}(k)$. Note that in our case, the effect of the term represented by Fig. 1(c) in Ref. [6] is absorbed by (b).

$$
\begin{gathered}
{\left[R_{+}^{p}, R_{-}^{q}\right]=\left[\delta^{p q} \sqrt{2 N /(N-1)} T^{D}+2\left(S^{m}\right)_{q p} S^{m}\right]} \\
{\left[R_{ \pm}^{p}, T^{D}\right]=\mp \sqrt{N / 2(N-1)} R_{ \pm}^{p}}
\end{gathered}
$$

Using the above basis, we can decompose the gauge field as

$$
\begin{aligned}
& Q_{\mu}^{a} T^{a}=Q_{\mu}^{m} S^{m}+\left(V_{-}^{p}\right)_{\mu} R_{+}^{p}+\left(V_{+}^{p}\right)_{\mu} R_{-}^{p}+Y_{\mu} T^{D}, \\
& A_{\mu}^{a} T^{a}=A_{\mu}^{m} S^{m}+\left(W_{-}^{p}\right)_{\mu} R_{+}^{p}+\left(W_{+}^{p}\right)_{\mu} R_{-}^{p}+Z_{\mu} T^{D} .
\end{aligned}
$$

Similarly, let us separate $\widetilde{\phi}$ into

$$
\widetilde{\phi}=\left(\begin{array}{l}
\phi \\
0
\end{array}\right)+\left(\begin{array}{c}
0 \\
\frac{1}{\sqrt{2}}\left(f_{N}+i g_{N}\right)
\end{array}\right),
$$

where $\phi$ is an $N-1$ component column matrix.

From the symmetry argument, we see the vacuum polarization takes the form

$$
\pi_{\mu \nu}\left(p_{\alpha}\right)=\left(g_{\mu \nu} p^{2}-p_{\mu} p_{\nu}\right) \pi_{e}\left(p^{2}\right)+\varepsilon_{\mu \nu \lambda} p_{\lambda} \pi_{o}\left(p^{2}\right)
$$

It is straightforward to express the Lagrangian in terms of the fields introduced above. To calculate the one-loop contributions to the parity-odd part of the vacuum polarization in the unbroken sector, it suffices to know four Feynman rules, which we list in the appendix.

Because of the partial cancellation between the first term in Eq. (4) and the gauge-fixing term in Eq. (5), in the background field gauge there is no three-leg vertex involving both the Higgs field and the unbroken gauge fields. As a result, only the two Feynman diagrams in Fig. 1 contribute to $\pi_{o}\left(p^{2}\right)$, in contrast with Ref. [6].

The result in the background field Landau gauge $(\xi=0)$ is that $\pi_{o}\left(p^{2}\right)=\pi_{o}^{(\mathrm{I})}\left(p^{2}\right)+\pi_{o}^{(\mathrm{II})}\left(p^{2}\right)$. Here

$$
\begin{aligned}
\pi_{o}^{(\mathrm{I})}\left(p^{2}\right)= & {\left[4(N-1) \pi^{2} m\right] \int \frac{d^{3} k}{(2 \pi)^{3}}\left\{\frac{\left[k^{2} p^{2}-(k \cdot p)^{2}\right]\left[4 m^{2}+10 k^{2}-10 k \cdot p+8 p^{2}\right]}{p^{2} k^{2}\left(k^{2}+m^{2}\right)(k-p)^{2}\left[(k-p)^{2}+m^{2}\right]}\right\} } \\
& +\left[4(N-1) \pi^{2} m\right] \int \frac{d^{3} k}{(2 \pi)^{3}}\left\{\frac{\left[-2 k^{2} p^{2}-2(k \cdot p)^{2}+4 p^{2}(k \cdot p)\right]}{p^{2} k^{2}\left(k^{2}+m^{2}\right)(k-p)^{2}}\right\},
\end{aligned}
$$

with $m \equiv \kappa g^{2} / 4 \pi$, and 


$$
\begin{aligned}
\pi_{o}^{(\mathrm{II})}\left(p^{2}\right)= & {\left[4 \pi^{2} m\right] \int \frac{d^{3} k}{(2 \pi)^{3}}\left\{\frac{\left[k^{2} p^{2}-(k \cdot p)^{2}\right]}{p^{2}\left(k^{2}+m_{+}^{2}\right)\left(k^{2}+m_{-}^{2}\right)\left[(k-p)^{2}+m_{+}^{2}\right]\left[(k-p)^{2}+m_{+}^{2}\right]}\right\} } \\
& \times\left\{6 m^{2}+\frac{\left(k^{2}+m_{+} m_{-}\right)\left[-m^{2}+8 k^{2}-4 k \cdot p+4 p^{2}\right]}{k^{2}}+\frac{\left[(k-p)^{2}+m_{+} m_{-}\right]\left[-m^{2}+8 k^{2}-12 k \cdot p+8 p^{2}\right]}{(k-p)^{2}}\right. \\
& \left.+\frac{\left(k^{2}+m_{+} m_{-}\right)\left[(k-p)^{2}+m_{+} m_{-}\right]\left[-6 k^{2}+6 k \cdot p-4 p^{2}\right]}{k^{2}(k-p)^{2}}\right\} \\
& +\left[4 \pi^{2} m\right] \int \frac{d^{3} k}{(2 \pi)^{3}}\left\{\frac{-2(k \cdot p)\left[m_{+} m_{-}+2 k^{2}-2 p^{2}\right]}{p^{2}\left(k^{2}+m_{+}^{2}\right)\left(k^{2}+m_{-}^{2}\right)(k-p)^{2}}+\frac{\left(k^{2}+m_{+} m_{-}\right)\left[-2 k^{2} p^{2}-2(k \cdot p)^{2}+4 k^{2}(k \cdot p)\right]}{p^{2} k^{2}\left(k^{2}+m_{+}^{2}\right)\left(k^{2}+m_{-}^{2}\right)(k-p)^{2}}\right\},
\end{aligned}
$$

with $m_{ \pm} \equiv \sqrt{m_{\mathrm{w}}^{2}+m^{2} / 4} \pm m / 2$, and $m_{\mathrm{w}} \equiv 1 / 2 g v$. In Eqs. (11) and (12), both the first integrals are identical to those obtained in the usual Landau gauge [6]. On the other hand, the second integral in Eq. (11) compensates for the effect of Fig. 2(a) while the second integral in Eq. (12) compensates for the effect of Figs. 1(c) and 2(b) there.

Carry out the integration explicitly, and we have

$$
\pi_{o}^{(\mathrm{I})}\left(p^{2}\right)=\frac{(N-1) \kappa}{4|\kappa|}\left[-1+\frac{\pi}{2} \hat{p}-\frac{3 \pi}{4} \hat{p}^{3}+\left(\hat{p}^{2}+1\right)\left(3 \hat{p}^{2}-1\right) \frac{1}{\hat{p}} \arctan \hat{p}-\frac{3}{2}\left(\hat{p}^{2}+4\right)\left(\hat{p}^{2}-2\right) \frac{1}{\hat{p}} \arctan \frac{\hat{p}}{2}\right],
$$

where $p \equiv \sqrt{p^{2}}$, and $\hat{p} \equiv p / m$.

$$
\begin{aligned}
\pi_{o}^{(\mathrm{II})}\left(p^{2}\right)= & \frac{\kappa}{4|\kappa|}\left(\frac{-2\left(m_{+}-m_{-}\right)}{m_{+}+m_{-}}+\frac{\pi p\left(m_{+}-m_{-}\right)\left(-2 m_{+} m_{-}+p^{2}\right)}{4 m_{+}^{2} m_{-}^{2}}\right. \\
& +\left\{\frac{\left[m_{+}^{2}\left(m_{+}-m_{-}\right)+\left(m_{+}+m_{-}\right) p^{2}\right]\left[m_{+}\left(m_{+}-2 m_{-}\right)+p^{2}\right]}{m_{+}^{2} m_{-}\left(m_{+}+m_{-}\right) p}\right\} \arctan \frac{p}{m_{+}} \\
& -\left\{\frac{\left[m_{-}^{2}\left(m_{+}-m_{-}\right)-\left(m_{+}+m_{-}\right) p^{2}\right]\left[m_{-}\left(2 m_{+}-m_{-}\right)-p^{2}\right]}{m_{+} m_{-}^{2}\left(m_{+}+m_{-}\right) p}\right\} \arctan \frac{p}{m_{-}} \\
& \left.+\left\{\frac{\left(3 m_{+}+m_{-}\right)\left[2 m_{+}\left(m_{+}+m_{-}\right)-p^{2}\right]\left(4 m_{+}^{2}+p^{2}\right)}{2 m_{+}^{2}\left(m_{+}+m_{-}\right)^{2} p}\right\} \arctan \frac{p}{2 m_{+}}\right\} \arctan \frac{p}{2 m_{-}} \\
& \left.-\left\{\frac{\left(m_{+}+3 m_{-}\right)\left[2 m_{-}\left(m_{+}+m_{-}\right)-p^{2}\right]\left(4 m_{-}^{2}+p^{2}\right)}{2 m_{-}^{2}\left(m_{+}+m_{-}\right)^{2} p}\right\} \arctan \left[\frac{p}{m_{+}+m_{-}}\right]\right) \\
& -\left\{\frac{\left(m_{+}-m_{-}\right)\left[\left(m_{+}+m_{-}\right)^{2}+p^{2}\right]\left[\left(m_{+}-m_{-}\right)^{2}+p^{2}\right]}{2 m_{+} m_{-}\left(m_{+}+m_{-}\right)^{2} p}\right]
\end{aligned}
$$

A first check on Eqs. (13) and (14) is to look at the $v=0$ limit (no breakdown of gauge symmetry). In this case, $\pi_{o}^{(\mathrm{II})}\left(p^{2}\right)$ becomes

$$
\pi_{o}^{(\mathrm{II})}\left(p^{2}\right) \stackrel{v \rightarrow 0}{\rightarrow} \frac{\kappa}{4|\kappa|}\left[-1+\frac{\pi}{2} \hat{p}-\frac{3 \pi}{4} \hat{p}^{3}+\left(\hat{p}^{2}+1\right)\left(3 \hat{p}^{2}-1\right) \frac{1}{\hat{p}} \arctan \hat{p}-\frac{3}{2}\left(\hat{p}^{2}+4\right)\left(\hat{p}^{2}-2\right) \frac{1}{\hat{p}} \arctan \frac{\hat{p}}{2}\right],
$$

and $\pi_{o}\left(p^{2}\right)$ is

$$
\begin{aligned}
\pi_{o}\left(p^{2}\right)= & \pi_{o}^{(\mathrm{I})}\left(p^{2}\right)+\pi_{o}^{(\mathrm{II})}\left(p^{2}\right) \\
& \rightarrow 0 \\
\rightarrow & \frac{N}{4} \frac{\kappa}{|\kappa|}\left[-1+\frac{\pi}{2} \hat{p}-\frac{3 \pi}{4} \hat{p}^{3}+\left(\hat{p}^{2}+1\right)\left(3 \hat{p}^{2}-1\right)\right. \\
& \left.\times \frac{1}{\hat{p}} \arctan \hat{p}-\frac{3}{2}\left(\hat{p}^{2}+4\right)\left(\hat{p}^{2}-2\right) \frac{1}{\hat{p}} \arctan \frac{\hat{p}}{2}\right] .
\end{aligned}
$$

Equation (16) is in agreement with the one-loop result obtained in Ref. [9] in a theory that is perturbatively equivalent to the Yang-Mills-Chern-Simons theory. We can also see that, when $v=0$,

$$
\pi_{o}\left(p^{2}\right) \stackrel{p \rightarrow 0}{\rightarrow} N \frac{\kappa}{|\kappa|}
$$

Next we shall consider the $p \rightarrow 0$ limit when $v \neq 0$. We found that, in this situation, 


$$
\begin{gathered}
\pi_{o}^{(\mathrm{II})}\left(p^{2}\right) \rightarrow 0, \\
\pi_{o}^{(\mathrm{I})}\left(p^{2}\right) \stackrel{p \rightarrow 0}{\rightarrow}(N-1) \frac{\kappa}{|\kappa|},
\end{gathered}
$$

and

$$
\pi_{o}(0)=\pi_{o}^{(\mathrm{I})}(0)+\pi_{o}^{(\mathrm{II})}(0)=(N-1) \frac{\kappa}{|\kappa|} .
$$

Again this in agreement with a calculation by Chen et al. [6] and Khare et al. [7], whose motivation was to check if the quantization condition is obeyed by the quantum corrections. (It can be pointed out that the result in Refs. [6] and [7] is obtained in the ordinary Landau gauge, so the agreement in two different gauges is not trivial because $\pi_{o}(0)$ is an offshell quantity [9]. This may be an indication that the topological constraint is working.) Equations (17) and (20) might lead one to believe that even if we set $\kappa$, the bare ChernSimons coefficient, to zero, an induced Chern-Simons term would survive, and SPB occurs in a perturbative way. However, a careful examination shows that, irrespective of whether or not $v$ is equal to 0 ,

$$
\pi_{o}^{(\mathrm{I})}\left(p^{2}\right) \stackrel{\kappa \rightarrow 0}{\rightarrow} 0, \quad \pi_{o}^{(\mathrm{II})}\left(p^{2}\right) \stackrel{\kappa \rightarrow 0}{\rightarrow} 0 .
$$

(The fact that $\pi_{o}^{(\mathrm{I})}\left(p^{2}\right) \stackrel{\kappa \rightarrow 0}{\rightarrow} 0$ was already noticed in Ref. [9].) Therefore, if we set $\kappa$ to zero first, there will be no induced parity-violating term.

What happens if the background gauge fields are in the broken sector? It is easy to see that the one-loop contributions to the parity-odd part of the proper self-energy also vanish if $\kappa$ is set to zero first because (1) the contributions from diagrams with one internal gluon (whether in the unbroken or broken sector) loop vanish in the same way as in the case considered above, and (2) for the diagram with one Higgs propagator and one gluon propagator, the contribution vanishes for the reason Khlebnikov [11] already gave in his first paper proposing SPB in the three-dimensional Abelian scalar electrodynamics, as we do not go to the $g=\infty$ limit. (Khlebnikov showed that SPB occurs when there is no treekinetic term for the Abelian gauge fields, i.e., when the Maxwell coupling parameter $e$ is infinite. When $e$ is finite, there is no SPB.)

The conclusion is that there is no basis to think that SPB can be detected in a perturbative study. It goes without saying that SPB may still happen in a nonperturbative manner. Presumably, one can proceed in the fashion of Nambu-JonaLasinio [12] or construct the effective potential for an appropriate order parameter and carry out a nonperturbative analysis [13]. This has not yet been done.

The work of Y.-C.K. and L.-Y.L. was supported by the National Science Council of the Republic of China under Grant No. NSC83-0208-M-002-022, and that of H.-C.K. under Grant No. NSC85-2112-M-001-004.

\section{Appendix}
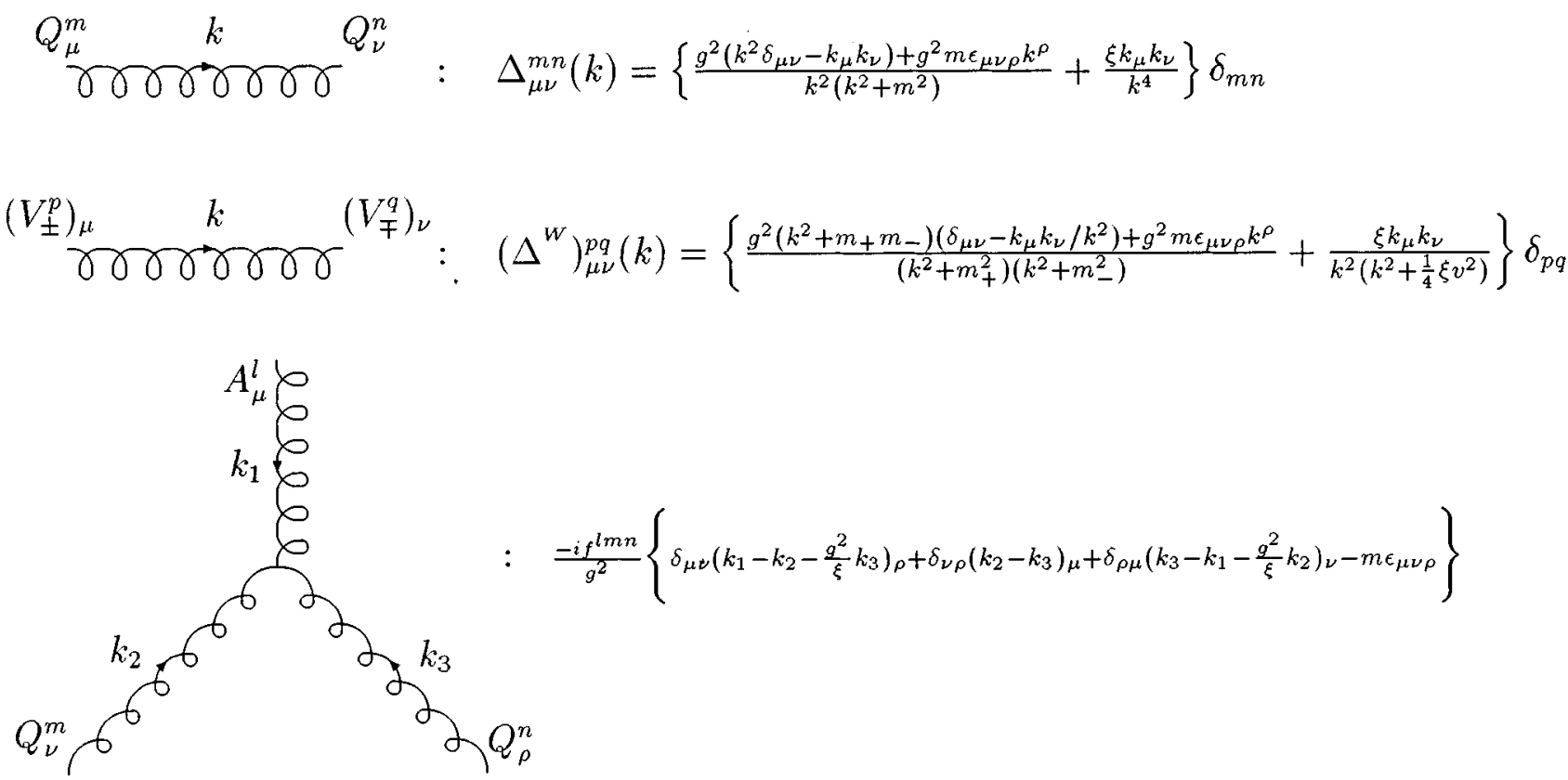


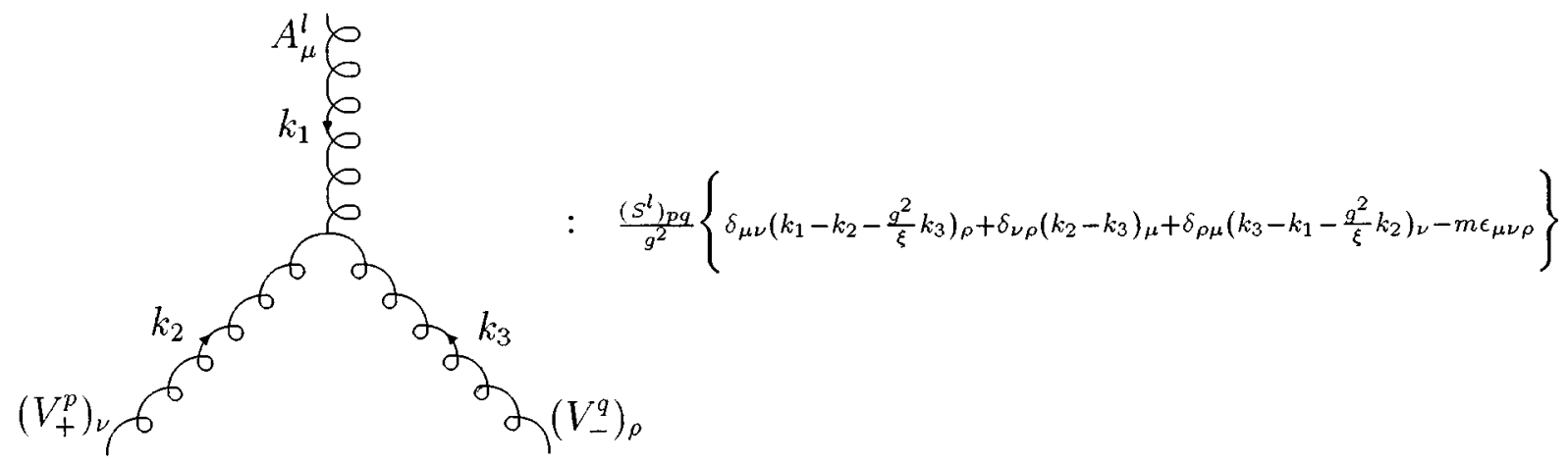

[1] W. Siegel, Nucl. Phys. B156, 135 (1979); J. F. Schonfeld, ibid. B185, 157 (1981); R. Jackiw and S. Templeton, Phys. Rev. D 23, 2291 (1981).

[2] S. Deser, R. Jackiw, and S. Templeton, Ann. Phys. (N.Y.) 140, 372 (1982).

[3] R.D. Pisarski and S. Rao, Phys. Rev. D 32, 2081 (1985).

[4] G. Giavarini, C.P. Martin, and F. Ruiz Ruiz, Nucl. Phys. B381, 222 (1992); L. Alvarez-Gaume, J. M. Labastida, and A. V. Ramallo, ibid. B334, 103 (1990); Y.-C. Kao and H.-N. Li, Can. J. Phys. 73, 344 (1995).

[5] S. Yu. Khlebnikov and M. E. Shaposhnikov, Phys. Lett. B 254, 148 (1991).

[6] L. Chen, G. Dunne, K. Haller, and E. Lim-Lombridas, Phys. Lett. B 348, 468 (1995).

[7] A. Khare, R. B. MacKenzie, P. K. Panigrahi, and M.B. Paran- jape, Phys. Lett. B 355, 236 (1995).

[8] L. F. Abbot, Nucl. Phys. B185, 189 (1981), and references therein.

[9] Y.-C. Kao, J. Koller, and H. Yamagishi, Phys. Rev. Lett. 58, 1077 (1987).

[10] K. Lee, Phys. Rev. Lett. 66, 553 (1991).

[11] S. Yu. Khlebnokov, JETP Lett. 51, 81 (1990).

[12] Y. Nambu and G. Jona-Lasinio, Phys. Rev. 122, 345 (1961).

[13] There is an interesting work by C. Vafa and E. Witten, Phys. Rev. Lett. 53, 535 (1984), proving that parity is not spontaneously broken in vector-like gauge theories. Khlebnikov and Shaposshnikov argued that the Vafa-Witten theorem does not apply here because of the noninvariance of the Chern-Simons term under large gauge transformations. 